\title{
Older Nurses' Perceptions of an Electronic Medical Record Implementation
}

\author{
Shaluni TISSERA ${ }^{\mathrm{a}, 1}$, Rebecca JEDWAB ${ }^{\mathrm{a}, \mathrm{b}}$, Rafael CALVO ${ }^{\mathrm{c}}, \mathrm{Naomi}_{\text {DOBROFF }}^{\mathrm{a}, \mathrm{b}}$, \\ Nicholas GLOZIER $^{\mathrm{d}}$, Alison HUTCHINSON ${ }^{\mathrm{a}, \mathrm{e}}$, Michael LEITER ${ }^{\mathrm{a}}$, \\ Elizabeth MANIAS ${ }^{\mathrm{a}}$, Katrina NANKERVIS ${ }^{\mathrm{a}, \mathrm{b}}$, \\ Helen RAWSON ${ }^{\mathrm{f}}$ and Bernice REDLEY ${ }^{\mathrm{a}, \mathrm{e}}$ \\ ${ }^{a}$ Deakin University, Melbourne, Victoria, Australia \\ ${ }^{\mathrm{b}}$ Monash Health, Melbourne, Victoria, Australia \\ ${ }^{\mathrm{c}}$ Dyson School of Design Engineering, Imperial College London, \\ London South, Kensington, United Kingdom \\ ${ }^{\mathrm{d}}$ The University of Sydney, Sydney, New South Wales, Australia \\ ${ }^{\mathrm{e}}$ Centre for Quality and Patient Safety Research - Monash Health \\ Partnership, Melbourne, Victoria, Australia \\ ${ }^{\mathrm{f}}$ Monash University, Melbourne, Victoria, Australia
}

\begin{abstract}
In Australia, almost $40 \%$ of nurses are aged 50 years and older. These nurses may be vulnerable to leaving the workforce due to challenges experienced during electronic medical record (EMR) implementations. This research explored older nurses' perceptions of factors expected to influence their adoption of an EMR, to inform recommendations to support implementation. The objectives were to: 1) measure psychological factors expected to influence older nurses' adoption of the EMR; and 2) explore older nurses' perceptions of facilitators and barriers to EMR adoption. An explanatory sequential mixed methods design was used to collect survey and focus group data from older nurses, prior to introducing an EMR system. These nurses were highly engaged with their work; $79.3 \%$ reported high wellbeing scores. However, their motivation appeared to be predominantly governed by external rather than internal influences. Themes reflecting barriers to EMR and resistance to adoption emerged in the qualitative data.
\end{abstract}

Keywords. Electronic medical record, older nurses, electronic medical record adoption, electronic medical record implementation

\section{Introduction}

Electronic medical records (EMR) provide a digital substitute to traditional paper-based medical records in hospitals. Success of EMR implementation relies on nurse engagement because they are the largest healthcare workforce in hospitals. Adoption of a new EMR system into the workplace can increase job demands on nurses [1] and reduce perceptions of control over their role and working conditions. Literature also suggests introducing an EMR may negatively affect nurses' workplace wellbeing [2] and contribute to worker burnout [3].

\footnotetext{
${ }^{1}$ Corresponding Author, Shaluni Tissera; E-mail: shalunitissera@gmail.com.
} 
Currently, two out of five Australian nurses and midwives are aged 50 years and over [4]. Studies show that worker performance and functional capacity can begin to decline after the age of 45 years [5], suggesting that some older nurses may experience greater difficulty than their younger counterparts in adapting to the changes associated with using a new EMR in their workplace [6]. As a consequence, older nurses may be vulnerable to leaving the workplace when an EMR is implemented. Replacement of older, knowledgeable nurses is costly, often exceeding the estimated $\$ 64,000$ turnover costs for a medical/surgical nurse [7]. Retaining older nurses in the workforce has many advantages [4]; they are among the most productive and reliable employees who contribute high levels of experience, skills, commitment and loyalty [8] as well as high professional competency [9]. While the benefits of retaining older nurses are well acknowledged [4], these nurses may need specific or tailored support in times of major change, such as implementing a new EMR [10].

Nurses' motivation is crucial for adopting new behaviours and adapting to challenging work circumstances within healthcare [11]. Poor adaptation to change is also impacted by burnout, which weakens overall work performance [2] and increases job turnover [12]. In addition, nurse engagement in the implementation of EMR systems has had a positive impact on post-implementation satisfaction and recognition by nurses that the EMR can enhance their practice [13].

The Theoretical Domains Framework (TDF) [14] provides a useful theoretical lens to examine facilitators and barriers to the adoption of an EMR by older nurses, and make recommendations to support effective change. The aims of this study were to: 1) measure psychological factors expected to influence older nurses' adoption of EMR; and 2) explore older nurses' perceptions of facilitators and barriers to EMR adoption.

\section{Methods}

Using an explanatory sequential mixed methods design, surveys and focus group data were collected from older nurses from five hospitals across a single large metropolitan health service in Victoria, Australia, prior to introducing an EMR. Low-risk ethics approval was obtained from both the health service and the university.

An organisation-wide cross-sectional survey was conducted in 2019. The survey included validated tools to measure nurse wellbeing (WHO-5 Wellbeing Index) [15], burnout (Maslach Burnout Inventory - General Scale (MBI-GS)) [16], EMR engagement (Autonomy and Competence in Technology Adoption (ACTA)) [17], work engagement (Utrecht Work Engagement Scale-3 (UWES-3)) [18] and satisfaction (global measures) [19].

Recruitment involved an email, containing an online link to the survey, sent by nurse managers to the 768 older nurses (aged 50 years and older) who were working in the wards where an EMR was to be implemented. Researchers visited the wards to provide information about the study and offer survey access online via a QR code or paper surveys as preferred. Survey data were entered into REDCap software, and IBM Statistics SPSS C software (V24) was used for statistical analysis. Survey tools were scored using the authors' instructions. Descriptive and inferential statistical tests were undertaken.

Four semi-structured focus groups and four individual interviews used a semistructured guide to collect qualitative data from a convenience sample of 21 older nurses. Demographic data were collected at commencement and all interviews were audio- 
recorded and transcribed verbatim for analysis [20]. Detailed field notes were also taken by an observer to assist analysis. Inductive coding used six steps of thematic analysis [21] and subsequent deductive content analysis involved mapping coded data to the 14 domains of the TDF [14]. All analyses were verified by at least two independent researchers.

\section{Results}

Eighty six nurses (11.2\% response rate) participated in the survey (aged between 50 and 66 years) and 21 nurses participated in focus groups or individual interviews (aged between 50 and 63 years). Participants' mean age and mean years of experience were similar across survey and interview participants. While over half $(54.7 \%, n=47)$ of participants reported high intention to stay, only $20 \%$ indicated they were very satisfied with their jobs (9-10/10), and another 20\% (7-8/10) were neutral. While $20.7 \%(n=18)$ reported wellbeing scores $(<50 / 100)$ indicating depression [27], the older nurses reported low burnout on subscales for exhaustion ( $\mathrm{M}=1.85$, SD 1.15) and professional efficiency $(\mathrm{M}=1.63, \mathrm{SD}=1.23)$, but moderate burnout on cynicism $(\mathrm{M}=1.45, \mathrm{SD}=1.08)$. Overall work engagement scores were high $(\mathrm{M}=4.124, \mathrm{SD}=1.02)$, supported by high dedication $(\mathrm{M}=4.43, \mathrm{SD}=1.13)$ and absorption $(\mathrm{M}=4.4, \mathrm{SD}=1.22)$, but moderate vigor $(\mathrm{M}=3.55$, $\mathrm{SD}=1.21)$ at work. These findings suggest older nurses were engaged, active, took initiative and generated their own positivity [18]. Scoring of the ACTA tool revealed older nurses' controlled regulation score (3.5) was higher than their autonomous regulation score (3.3) resulting in a negative Relative Autonomous Index (-0.20). This finding indicates older nurses' motivation to adopt the EMR was predominantly extrinsic, governed by high control and low autonomy [17]. Tool reliability measures using Cronbach alpha were acceptable for all scales and subscales $(>.78)$.

Inductive thematic analysis of the qualitative interview data revealed three themes as outlined below:

1. Hopeful improvement of work-life encapsulated older nurses' uncertainty about the EMR implementation as an enhancement to nursing practice and their profession. The majority of the data supported this theme and nurses displayed an imprecise self- understanding about the impact of EMR adoption on their clinical practice. Three sub- themes were: i) Benefits to practice highlighted older nurses' positive perceptions of the EMR: "I think it's going to be beneficial for all...it's going to actually save a lot of time”; ii) Reserved judgment reflected nurses' ambivalence regarding the EMR: "I'm just waiting to see"; iii) Resisting change illustrated barriers to accepting the EMR: "We are very busy, because there's so much that we do".

2. What I need for self-determination highlighted how to tailor EMR implementation for theses nurses. Two sub-themes were: i) Skills reflected older nurses' self-identified ability and competence to using new technology such as the EMR: "How do we get into that? Because it's not something that we grew up with"; ii) Resources reflected the older nurses' suggestions and needs regarding education, training and ongoing support: "It's not only a lot of education, also lot of support".

3. External influences explained older nurses' perceptions of how organisational factors and peer pressure affected their EMR adoption: "Sometimes you can't change things...I sort of just accept whatever it is that we've got at the moment 
with...the system". Mapping of coded data to the TDF revealed facilitators and barriers to EMR adoption related to 12 of the 14 domains; $58 \%$ linked to barriers. Knowledge $(12 \%)$ and optimism $(7.2 \%)$ emerged as the most common facilitators, whilst the most common barriers related to environmental context and resources (13.5\%) and beliefs about consequences (11.9\%). Reinforcement and intentions were not identified in the data.

\section{Discussion}

Before EMR implementation, older nurses reported low job satisfaction and low intention to stay in their workplace, despite also reporting high levels of wellbeing and work engagement. The findings of predominantly negative perceptions of the EMR, and frequent identification of barriers to EMR implementation contradict previous literature [22]. This data suggests the loss of older nurses in the workplace is a real risk during an EMR implementation. Losing these skilled and experienced older nurses would have detrimental effects on the workplace, patient safety outcomes and large financial implications [7].

Older nurses reported high levels of work engagement and low levels of burnout in their current practice, similar to previous literature [23]. High scores for intrinsic motivation $(\mathrm{M}=3.20, \mathrm{SD}=1.0)$ were supported by qualitative comments, indicating they were looking forward to using the EMR, and interested in adopting the EMR, despite fears about using the new technology and challenges they perceived would occur. However, data also indicated that older nurses' motivation was dominated by external factors such as organisational pressure, performance expectations and social influences imposed on them in the workplace, more so than exercising choice or autonomy [17].

Barriers to EMR adoption dominated older nurses' perceptions of the EMR; most barriers related to perceptions of the environmental context and resources, and beliefs about consequences. Specific barriers related to pre-conceived judgment, a 'patients first' attitude and negative emotions. In addition, and similar to previous studies, the elements of training, education, support, resources, skills, clinical documentation, clinical role, teamwork, expected clinical practice and the technological advancement in healthcare were identified as both facilitators and barriers [24].

The predominantly negative perceptions about EMR may be explained in part by the mismatch between perceptions of the strategies being used for implementation (i.e. high control) and the preferred working styles of older, experienced nurses (i.e. high autonomy). It is possible that older nurses' views could worsen post-EMR implementation, [25] and hence, contribute to them leaving the workforce. Understanding nurses' perceptions of barriers can be used to develop tailored strategies to support effective change management. For example, build intrinsic motivation using persuasion and incentivisation or training and education tailored to individual roles [14]. Six elements of the TDF were related to older nurses' perceptions of facilitators to EMR adoption: efficiency, safety, access, supportive work environment, past experience on computer systems, and a 'can do' attitude. Specifically, older nurses identified the need for unrestricted access to a prototype, hands-on experience, continuous training and ongoing pre and post-implementation support.

Strengths of this study included use of reliable and valid tools, and comparison of findings with current literature [26]. While the survey response rate was low $(11.2 \%)$, participants in focus groups and individual interviews had similar demographics and 
included diverse representation of older nurses. These findings provide the foundation for future investigation of factors impacting older nurses' adoption of EMR.

\section{Conclusions}

Change associated with impending EMR implementation was viewed as a significant challenge for older nurses and may therefore pose a risk to their retention in the workforce. Specific strategies to address technology anxiety as well as the physical and psychological limitations of an older workforce are needed to support these nurses during an EMR implementation.

\section{Acknowledgements}

The authors would like to acknowledge the healthcare organisation and research participants.

\section{References}

[1] Topaz M, Ronquillo C, Peltonen LM et al. Nurse informaticians report low satisfaction and multi-level concerns with electronic health records: Results from an International Survey. Annual Symposium proceedings. AMIA Symposium 2016 (2017), 2016-2025.

[2] Cramer E, Hunter B Relationships between working conditions and emotional wellbeing in midwives. Women and Birth 32 (2018), 521-532.

[3] El-Jardali F, Dimassi H, Dumit N, Jamal D, Mouro G. A national cross-sectional study on nurses' intent to leave and job satisfaction in Lebanon: Implications for policy and practice. BMC Nursing 8 (2009), 3.

[4] Australian Institute of Health and Welfare Australia's health 2018: In brief. Australian Government, 2018.

[5] Soer R, Brouwer S, Geertzen JH, van der Schans CP, Groothoff JW, Reneman MF Decline of functional capacity in healthy aging workers. Archives of Physical Medicine and Rehabilitation 93 (2012), 23262332.

[6] Fragar LJ, Depczynski JC Beyond 50. Challenges at work for older nurses and allied health workers in rural Australia: A thematic analysis of focus group discussions. BMC health services research 11 (2011), 42-42.

[7] Roche MA, Duffield CM, Homer C, Buchan J, Dimitrelis S. The rate and cost of nurse turnover in Australia. Collegian 22 (2015), 353-358.

[8] Childre F, Mangino M. The aging employee: Impact on occupational health. Workplace Health \& Safety Journal 48 (2000), 349-359.

[9] Costa G, Sartori S. Ageing, working hours and work ability. Ergonomics 50 (2007), 1914-1930.

[10] Keller SM, Burns CM. The aging nurse: Can employers accommodate age-related changes? Workplace Health \& Safety Journal 58 (2010), 437-446.

[11] Kocabas I. The effects of sources of motivation on teachers' motivation levels Education 129 (2009), 724-734.

[12] Price RH, Hooijberg R. Organizational exit pressures and role stress: Impact on mental health. Journal of Organizational Behavior 13 (1992), 641-651.

[13] Huryk LA. Factors influencing nurses' attitudes towards healthcare information technology. Journal of Nursing Management 18 (2010), 606-612.

[14] Atkins L, Francis J, Islam R et al. A guide to using the Theoretical Domains Framework of behaviour change to investigate implementation problems. Implementation Science 12 (2017), 1-18.

[15] Topp CW, Østergaard SD, Søndergaard S, Bech P. The WHO-5 Well-Being Index: A systematic review of the literature. Psychotherapy and Psychosomatics 84 (2015), 167-176.

[16] Maslach C, Jackson S, Leiter M. The Maslach Burnout Inventory Manual. In, Consulting Psychologists Press, Palo Alto, CA, 1997, pp. 191-218. 
[17] Peters D, Calvo RA, Ryan RM. Designing for motivation, engagement and wellbeing in digital experience. Frontiers in Psychology 9 (2018).

[18] Schaufeli WB, Shimazu A, Hakanen J, Salanova M, De Witte H. An ultra-short measure for work engagement: The UWES-3 validation across five countries. European Journal of Psychological Assessment (2017), No Pagination Specified-No Pagination Specified.

[19] Saari LM, Judge TA. Employee attitudes and job satisfaction. Human Resource Management 43 (2004), 395-407.

[20] Krueger RA, Casey MA. Designing and conducting focus group interviews. In, St Paul, Minnesota, USA, 2002.

[21] Braun V, Clarke V. Using thematic analysis in psychology. Qualitative research in psychology 3 (2006), 77-101.

[22] Ingersoll GL, Olsan T, Drew-Cates J, DeVinney BC, Davies J. Nurses' job satisfaction, organizational commitment, and career intent. JONA: The Journal of Nursing Administration 32 (2002), 250- 263.

[23] Maslach C, Schaufeli WB, Leiter MP. Job burnout. Annual review of psychology 52 (2001), $397-422$.

[24] Miller RH, Sim I. Physicians' use of electronic medical records: Barriers and solutions. Health affairs 23 (2004), 116-126.

[25] Laramee AS, Bosek M, Shaner-McRae H, Powers-Phaneuf T. A comparison of nurse attitudes before implementation and 6 and 18 months after implementation of an electronic health record. CIN: Computers, Informatics, Nursing 30 (2012), 521-530.

[26] Boynton PM, Greenhalgh T. Selecting, designing, and developing your questionnaire. British Medical Journal 328 (2004), 1312-1315. 